\title{
The multiplicative effect of combining alcohol with energy drinks on adolescent gambling
}

Alessio Vieno, PhD, ${ }^{\mathrm{a}}$ Natale Canale, $\mathrm{PhD},{ }^{\mathrm{a}}$ Roberta Potente, PhD, ${ }^{\mathrm{b}}$ Marco Scalese, PhD, ${ }^{\mathrm{b}}$ Mark D. Griffiths, PhD, ${ }^{\mathrm{c}}$ Sabrina Molinaro, $\mathrm{PhD},{ }^{\mathrm{b}}$

\author{
Affiliations: \\ ${ }^{a}$ Department of Developmental and Social Psychology, University of Padova, Italy; \\ ${ }^{\mathrm{b}}$ Institute of Clinical Physiology, National Council of Research, Italy; \\ c International Gaming Research Unit, Psychology Department, Nottingham Trent University, \\ United Kingdom
}

Address correspondence to: Sabrina Molinaro, Institute of Clinical Physiology, National Council of Research, Via Moruzzi 1, 56124 Pisa, Italy (sabrina.molinaro@ifc.cnr.it)

Short title: Alcohol mixed with energy drinks and gambling

Funding Source: This research did not receive any specific grant from funding agencies in the public, commercial, or not-for-profit sectors.

Conflict of Interest: All the authors apart from Dr. Griffiths have indicated they have no potential conflicts of interest to disclose. Dr. Griffiths has received funding for a number of research projects in the area of gambling education for young people, social responsibility in gambling and gambling treatment from the Responsibility in Gambling Trust, a charitable body which funds its research program based on donations from the gambling industry. Dr. Griffiths also carries out consultancy for the gambling industry in relation to responsible gambling, player protection and harm minimization.

Financial Disclosure: The authors have no financial relationships relevant to this article to disclose.

Word count (excluding title page, abstract, references, figures and tables): 3,690

Number of tables: 2

Number of figures: 2

Number of references: 48

Abbreviations: AmED, alcohol mixed with energy drinks; ARPG, at-risk and problem gambling; SOGS-RA, South Oaks Gambling Screen-Revised for Adolescents; ESPAD, European School Survey Project on Alcohol and Other Drugs; FDA, US Food and Drug Administration 


\begin{abstract}
Purpose. There has been increased concern about the negative effects of adolescents consuming a combination of alcohol mixed with energy drinks (AmED). To date, few studies have focused on AmED use and gambling. The present study analyzed the multiplicative effect of AmED consumption, compared to alcohol alone, on the likelihood of at-risk or problem gambling during adolescence.

Methods. Data from the ESPAD ${ }^{\circledR}$ Italia 2015 study, a cross-sectional survey conducted in a nationally representative sample of students (ages 15 to 19 years) were used to examine the association between self-reported AmED use ( $\geq 6$ times, $\geq 10$ times, and $\geq 20$ times during the last month) and self-reported gambling severity. Multivariate models were used to calculate adjusted prevalence ratios to evaluate the association between alcohol use, AmED use, and gambling among a representative sample of adolescents who reported gambling in the last year and completed a gambling severity scale $(n=4,495)$.

Results. Among the 19\% students classed as at-risk and problem gamblers, $43.9 \%$ were classed as AmED consumers, while $23.6 \%$ were classed as alcohol consumers (i.e. did not mix alcohol with energy drinks). In multivariate analyses that controlled for covariates, AmED consumers were three times more likely to be at-risk and problem gamblers $(\mathrm{OR}=3.05)$ compared to non-consuming adolescents, while the effect became less pronounced with considering those who consumed alcohol without the addition of energy drinks $(\mathrm{OR}=1.37)$.
\end{abstract}

\title{
Conclusions
}

The present study clearly established that consuming AmED might pose a significantly greater risk of experiencing gambling-related problems among adolescents.

Keywords: alcohol mixed with energy drinks, problem gambling, gambling, ESPAD, youths 
Over the last decade, a large number of products containing high levels of caffeine have emerged and have been disproportionally targeted towards adolescents and young adults. This has led to increased concern by pediatricians towards their potentially serious adverse effects on children and adolescents (Seifert, Schaechter, Hershorin, \& Lipshultz, 2011). Moreover, the sales of energy drinks grew 60\% between 2008 and 2013 and have coincided with increased reports of emergency department visits because of its consumption combined with that of alcohol (Bonar et al., 2015). For these reasons, there is a growing interest and concern about the use of alcohol mixed with energy drinks (AmED) especially among youth. Because of this, the US Food and Drug Administration (FDA) banned the sale of energy drinks premixed with alcohol in 2010 (FDA, 2010). However, individuals (and particularly adolescents) continue to mix energy drinks with alcohol. For instance, studies have shown that $23 \%$ to $47 \%$ of adolescents and young adult alcohol users consume alcohol-mixed energy drinks (Peacock, Pennay, Droste, Bruno, \& Lubman, 2014).

Examining this phenomenon, a review of the negative effect of AmED found that consuming alcohol mixed with energy drinks was more dangerous than consuming alcohol alone because of the stimulant effects of caffeine contained in energy drinks (Striley \& Khan, 2014). This stimulant effect might be perceived as offsetting the depressant effects of alcohol, resulting in adolescents feeling less intoxicated than they actually are, and described as "wide awake drunkenness" (Arria \& O'Brien, 2011). AmED-induced underestimation of intoxication has been found to be associated with various risky behaviors (such as alcohol and drug use, smoking, sexual risk-taking, and violence) among adolescents and young adults (Miller, 2008; Scalese et al., 2017).

However, AmED may also be associated with problem gambling. In the last decade, problem gambling among adolescents has emerged as an increasing social and public health issue (Molinaro et al., 2014). For instance in Italy, results from the European-School-Survey-Project-onAlcohol-and-Other-Drugs (ESPAD ®Italia) showed that during 2014, 39.3\% of high school students gambled at least once in the past 12 months (boys $=48.8 \%$; girls $=29.7 \%$ ) and $11.1 \%$ and $7.6 \%$ of these were classified as at risk and problem gamblers respectively (DAP, 2016). 
Problem gambling has been shown to have multiple-related risk factors similar to those of other addictive behaviors (Leeman \& Potenza, 2012). Given the similarity in the risk factors between gambling and alcohol and drug use and misuse, it is possible that the use of AmED is positively associated with gambling problems. According to Pennay and collegues (2015), the association between AmED use and problem gambling intuitively makes sense considering that caffeine in energy drinks might result in higher impulsivity before or during gambling session or also be used to prolong wakefulness and attention while gambling. Additionally, studies conducted in animals have suggested increased impulsive decision-making following acute caffeine administration (Diller, Saunders, \& Anderson, 2008; Flora \& Dietze, 1993). From this perspective, AmED may affect at-risk and problem gambling (ARPG).

Gamblers have a high tolerance toward risk (Brevers, Bechara, Cleeremans, \& Noël, 2013), and pathological gambling has been associated with alterations of dopaminergic regions linked to reward, risk, and motivation (Potenza, 2014). Furthermore, both alcohol and caffeine increase dopamine through the body's reward pathways via dopamine and adenosine receptors in the nucleus accumbens (NA) and the dorsal striatum (DS) (Fuxe, Ferré, Genedani, Franco, \& Agnati, 2007). The DS is involved in response control, decision-making, and habitual action whereas the NA is involved in learning and behaviors that are reward-associated, particularly drug abuse (Fuxe et al., 2007). Decreased striatal activation during reward processing has been found in gambling disorder, binge-eating, and alcohol dependence (Potenza, 2014). Repeated consumption of caffeine-mixed alcohol causes stronger activation of the dopaminergic reward pathway than caffeine or alcohol alone (Robins, Lu, \& van Rijn, 2016). Furthermore, previous studies examining neurobiological correlates of problem gambling have identified abnormalities in brain dopamine systems that are crucial for reward sensitivity (Wardell, Quilty, Hendershot, \& Bagby, 2015). Therefore, we hypothesized that this combined consumption could be connected to the propensity for ARPG. Thus, repeated co-consumption of energy drinks and alcohol may increase the probability of ARPG.

To date, few studies have focused on AmED use and gambling. More specifically, to the 
best of our knowledge only two studies have examined the association between AmED use and gambling, and both of these were among the adult population. In the first study, Pennay and colleagues (2015) found that odds of being an alcohol and energy drink (AED) consumer increased as a function of reporting moderate risk/problem gambling. The second study involved a community sample and reported that matched-frequency participants (AmED and alcohol use) reported significantly lower odds of spending more money than planned and gambling (Peacock et al., 2015). Based on the findings of these two studies, it appears that the relationship between AmED and gambling is inconsistent. The inconsistency might be due to methodological flaws and relevant differences in the population studied. For example, Pennay and colleagues (2015) used a measure of gambling severity (Problem Gambling Severity Index- Ferris \& Wynne, 2001), while Peacock and colleagues (2015) considered measures for financial outcomes during AmED sessions (i.e., 'spent more money than planned' and 'gambled'). In addition, one study described patterns of AmED use in a representative sample of the Australian population (Pennay et al., 2015), while the second used a convenience sample comprising a within-participant matched-frequency sample (i.e., participants who reported the same frequency of alcohol and AmED use). By using a large-scale nationally representative sample of Italian youth, the principal aim of the present study was to clarify the association between AmED use and adolescent ARPG. According to previous studies suggesting that there is a synergistic effect between stimulant use and alcohol use in combination (Arria \& O'Brien, 2011), it was hypothesized there would be a multiplicative effect of AmED consumption, compared to alcohol alone, among at-risk and problem gambling adolescents.

\section{METHODS}

\section{Study population}

The present study used the data from ESPAD ${ }^{\circledR}$ Italia 2015, a national school-survey conducted annually to monitor risk-behaviors among Italy's high school students, and is included in the largest cross-national research project ESPAD (European School Survey Project on Alcohol and 
Other Drugs). The survey is included in the Scholastic Plan for Education (Decree of the President of the Italian Republic n.275/1999, Art. 8), edited and approved by Collegial Bodies, including teachers, parents, and students (Legislative Decree n.297/1994). Multistage stratified sampling was used as sampling procedure. First, the provinces were stratified by geographical area (North, Central, South, and Islands) and by population density. Second, the schools were stratified by type of school and by geographical location (urban and rural). Finally, a number of schools were extracted from each stratum and within each school one or more sections were chosen, in which the questionnaire was administered from the first to the fifth class. Parental permission for their children to participate was obtained prior to survey administration. Students were informed that participation was anonymous and voluntary. The response rate of participating schools was $87.7 \%$. Self-administered questionnaires were completed by a representative sample of high school students, aged $15-19$ years, in school classrooms $(n=13,725)$. To study the variables associated with different types of gamblers (e.g., "non-problem” gamblers, "at risk" gamblers, "problem" gamblers), a subsample of 4,495 Italian high-school students who reported gambling in the last year and completed the South Oaks Gambling Screen-Revised for Adolescents (SOGS-RA) (Winters, Stinchfield, \& Fulkerson, 1993) was extracted from the dataset for further analysis (see Table 1 for the sample characteristics). Gambling was more prevalent among males and the sample comprised $62.5 \%$ male adolescents aged between 15 and 19 years.

\section{Measures}

\section{Dependent variable}

Gambling severity was assessed using the SOGS-RA (Winters et al., 1993; Colasante et al., 2014), comprising 12 dichotomous items (no=0; yes=1) assessing gambling behavior and gamblingrelated problems during the past 12 months. The total score ranges from 0 to 12 and provides three categories: non-problem gambler (scoring 0 to 1), at-risk gambler (scoring 2 to 3), and problem gambler (scoring 4 or above). A dichotomized variable was generated: non-problem gambler 
(NOPG) and at-risk/problem gambler (ARPG) (Walther, Morgenstern, \& Hanewinkel, 2012; Carbonneau, Vitaro, Brendgen, \& Tremblay, 2015). The instrument had adequate internal reliability $(\alpha=.85 ; 95 \% \mathrm{CI}=.84-.86)$. Additionally, a recent systematic review conducted by Edgren and colleagues (2016) found that most adolescent gambling studies used the SOGS-RA as the primary adolescent ARPG instrument.

\section{Independent variables - Beverage consumption}

The survey also comprised questions relating to student's alcohol consumption and their AmED consumption. The following questions were analyzed: (i) "During the last month, on how many occasions (if any) have you used alcohol mixed with Energy Drinks (AmED) (responses from 0 to 20 or more times in last month)" and (ii) "During the last month, on how many occasions (if any) have you used alcohol (responses from 0 to 20 or more times in last month). The following frequencies of consumption were used as cut off point of AmED and alcohol consumption in last month (LM): $\geq 6$ times; $\geq 10$ times; $\geq 20$ times. The present authors relied on cut-off values used in other studies, namely, moderate/heavy ED users when participants reported to consume (six or more times) an energy drink (e.g., Monster, Red Bull) during the past 30 days (students in the $8^{\text {th }}$, $10^{\text {th }}$, or $12^{\text {th }}$ grade) (Polack et al., 2016). Additionally, a published survey commissioned by the European Food Safety Authority (Zucconi, Volpato, Adinolfi, \& Gandini, 2013) defined "High chronic" ED consumers as adolescent who regularly consumed ED "4-5 times a week" or more (on average 16-20 times for four weeks). We therefore decided to apply a cut-off of 20 or more times for a chronic AmED consumption. Each type of consumption (alcohol alone and AmED) was coded $(\mathrm{No}=0$ and $\mathrm{Yes}=1)$ according to the cut-off point of LM frequencies ( $\geq 6$ times; $\geq 10$ times; and $\geq 20$ times). Finally, a single variable "Beverage consumption LM" was generated on the basis of the cut-off point of frequencies of type of consumption (alcohol alone and AmED) in LM and coded as:

- "No": No alcohol and No AmED consumption;

- "Alcohol alone": consumption of alcoholic beverage but not mixed with energy drinks; 
- “AmED": consumption of alcoholic beverage mixed with energy drinks.

Consequently, three separate categorical measures were used in analysis, each of which used different cut-offs for use ( $\geq 6$ times; $\geq 10$ times; and $\geq 20$ times). The primary cut-off used was $\geq 6$ times. Further analyses used the remaining cut-offs for use ( $\geq 10$ times; and $\geq 20$ times).

\section{Background characteristics}

Other variables included were:

- $\operatorname{Gender}($ Females $=0 ;$ Males $=1)$;

- Age $(15-17$ years $=0 ; 18-19$ years $=1)$ according to the Italian Gambling Law, that prohibits gambling under the age of 18 years;

- Level of parents' schooling, assessed by the question "What is the highest level of schooling your mother/father completed?". The coding was: "Completed primary school or less", "Some secondary school", "Completed secondary school", "Some college", "Completed college or some university", and "Completed university";

- Perceived economic family status, compared to other Italian family, was evaluated by the answer on a 7-point scale to the question "What is the economic status of your family compared to others?", from "Very much above" to "Very much below", coded in High (Very much above/Much above/Above), Medium (About the same), Low (Below/Much below/Very much below).

Perceived economic family status and average parental education were used to control for the home environment in which the adolescents developed (Steinberg, Mounts, Lamborn, \& Dornbusch, 1991).

\section{Statistical analyses}

The statistical analyses were carried out with SPSS software (version 23). A logistic regression analyses was used to test for the possible contribution of the use of both alcohol and 
AmED (controlling for sociodemographic characteristics) to ARPG. All confidence intervals were set at the $95 \%$ level. The steps taken in the regression analysis included: introducing the first main independent variable (the use of both alcohol and AmED) and then by adding the possible confounders (gender, age, parent's education, and perceived economic family status using a singlestep procedure). In the first stage (principal analyses), a cut of point of $\geq 6$ time for the use of both alcohol and AmED was used. Parallel analyses were conducted by using different cut-off points for the use of both alcohol and AmED ( $\geq 10$ time, and $\geq 20$ time). As it was beyond the scope of the present study to analyze the variance of ARPG across schools, no attempt was made to statistically account for school-level variances.

\section{RESULTS}

Descriptive statistics of all variables are presented in Table 1. The sample (high-school students who reported gambling in the last year) was $62.5 \%$ male and $52.1 \%$ of respondents were aged between 15 and 17 years. Across the sample, 5.1\% of respondents reported drinking AmED in the last month greater than or equal to 6 times. In the past-year gambler sample (total sample), $19.0 \%$ were at-risk and problem gamblers. Of the AmED consumers (greater than or equal to 6 times) in the past 30 days, $43.9 \%$ were classified as at-risk and problem gamblers, while $23.6 \%$ were classified as at-risk and problem gamblers among alcohol consumers (greater than or equal to 6 times) in the past 30 days (see Figure 1).

\section{[INSERT ABOVE HERE TABLE 1 AND FIGURE 1]}

Table 2 displays the results of the logistic regressions. The odds of ARPG were four times as high among adolescents who consumed AmED compared to non-consuming adolescents (odds ratios $(\mathrm{ORs})=4.19$ [95\% CI: 3.17-5.53]). Adding gender, age, parent's education, and perceived economic family status to the models only slightly weakened the association of AmED consumption with ARPG. More specifically, AmED consumers were three times more likely to be at-risk and problem gamblers $(\mathrm{OR}=3.05)$ compared to non-consuming adolescents, while the 
effect became less pronounced with considering those who consumed alcohol without the addition of energy drinks $(\mathrm{OR}=1.37)$. Boys were more likely to be at risk-problematic gamblers than girls. Among family socio-demographic characteristics, students who perceived their financial family status as 'medium' were significantly less likely than adolescents who perceived their financial family status as 'low' to be at-risk and problem gamblers. In addition, students with parents with higher parental educational degrees were significantly less likely than adolescents who lived with parents with lower parental educational degrees to be at-risk and problem gamblers.

\section{[INSERT ABOVE HERE TABLE 2]}

Parallel analyses were conducted with a more "restrictive" cut-off in the use of AmED and alcohol, including "greater than or equal to 10 times" and "greater than or equal to 20 times" in the last month. By increasing exposure to both alcohol and AmED, the association with ARPG increased (see Figure 2).

[INSERT ABOVE HERE FIGURE 2]

\section{DISCUSSION}

The present study is the first national prevalence survey to examine the rates of ARPG among adolescents who reported consuming AmED in the last month. The odds of ARPG were three times as high among adolescents who consumed AmED compared to adolescents not consuming AmED or alcohol. Moreover, by comparing this effect with alcohol consumption, the effect of AmED is over twice the one of consuming alcohol alone. This result is in line with a previous series of comprehensive literature reviews exploring the use of AmED and associated measures of consumption and harm outcomes (Arria \& O’Brien, 2011; McKetin, Coen, \& Kaye, 2015) extending them for the first time to the context of ARPG in youth.

The results of the present study are in line with the study conducted by Pennay and colleagues (2015) who found that odds of being an alcohol and energy drink consumer increased as a function of reporting moderate risk/problem gambling. There may be a few reasons why this relationship 
between AmED consumption and risky behaviors has now been identified with respect to adolescent ARPG. The first possible explanation refers to the self-reported psychological outcomes of AmED versus alcohol consumers. More specifically, Peacock and colleague (2014) found higher odds of feeling irritable and 'on edge', and lower odds of feeling calm, carefree, friendly, outgoing, and sociable, during AmED relative to alcohol sessions. This psychological profile might translate into more problem gambling given that adolescent problem gambling is associated with more psychological complaints (e.g., feeling depressed; being irritable or having a bad temper; feeling nervous) (Dussault, Brendgen, Vitaro, Wanner, \& Tremblay, 2011).

Second, previous studies have reported that caffeine and alcohol are known to increase dopamine release in dopaminergic reward pathways (Fuxe et al., 2007). Dopamine status is relevant to personality-related factors (e.g., sensation-seeking and impulsivity) implicated in the development of gambling disorder (Grant, Odlaug, \& Chamberlain, 2016). In fact, AmED consumers scored higher on impulsivity than alcohol-only consumers (Snipes, Jeffers, Green, \& Benotsch, 2015). Two recent meta-analyses have suggested that association of AmED use with risky behaviors can be interpreted in terms of phenotypical differences between AmED and alcohol without energy drink consumers, such as personality traits or propensity for risk-taking. Little research has examined impulsivity in the context of AmED consumption among adolescent and young people, but it is well established that adolescents with gambling problems have higher levels of impulsivity than non-problem gamblers (Derevensky \& Gilbeau, 2015; Canale, Scacchi, \& Griffiths, 2016). Additionally, previous studies have shown that repeated dopaminergic activation of prefrontal pathways by chronic stimulant use (e.g., alcohol mixed with caffeine) eventually impairs inhibitory functions, leading to a loss of control over behavioral impulses (Lyvers, 2000), which in turn is strongly associated with problem gambling (Blaszczynski \& Nower, 2002). As heavier drinking caffeinated alcoholic beverages can inhibit behavioral control (Marczinski, 2011), it is possible that the addition of caffeine increase an individual's impulsivity, resulting in impulsive behaviors, such as gambling. 
Third, gambling has long been considered a form of risk-taking, in that gambling necessarily comprises consistent exposure of one's monetary resources to outcome variance. In that respect, adolescent gambling can be understood as one potential risk behavior associated with AmED use for the following reason: adolescents with a high risk taking tendency, may be drawn to energy drinks, alcohol, and gambling because they are motivated by behaviors (such as AmED use and gambling) that provide stimulation and reward (Zuckerman, 1994).

Finally, a possible alternative explanation of the connection between AmED consumption and ARPG may be due to a general underlying vulnerability. Adolescents that are more prone to adopt risky behaviors (also including ARPG) might also have a tendency to drink alcohol in combination with energy drinks. For at least some of these problem behaviors, the association appears to be due to a higher general risk taking tendency among AmED consumers (Brache \& Stockwell, 2011). Therefore, AmED might be a part of a broader cluster of adverse behaviors (van Nieuwenhuijzen et al., 2009), because adolescents who are vulnerable to behaving in a risky manner prefer these types of activity.

With regard to demographic characteristics, being male was associated with higher levels of ARPG according to a recent meta-analysis (see Dowling et al., 2017) confirming that male gender is a risk factor that has concurrently been associated with gambling problems in cross-sectional studies of young people. In terms of family socio-demographic characteristics (parental education and perceived economic family status), the present study found that adolescents who perceived their financial family status as medium were significantly less likely than adolescents who perceived their financial family status as low to be at-risk and problem gamblers. In addition, adolescents who lived with parents with lower parental educational degrees were significantly more likely than adolescents who lived with parents with higher parental educational degrees to be at-risk and problem gamblers. Consistent with results from previous studies (e.g., Canale et al., 2017a-b; Hayer \& Griffiths, 2016; Welte, Barnes, Tidwell, \& Hoffman, 2008), the findings of the present study suggested that the higher scores of gambling severity in socio-economically disadvantaged youth 
may be explained by high levels of impulsivity in this youth. In fact, a previous longitudinal study found that the low parental education and high area material deprivation were associated with gambling onset among impulsive youth only (Auger, Lo, Cantinotti, \& O'Loughlin, 2010).

The present study also found that the likelihood of being an at-risk-problem gambler among students increases substantially with higher increases of monthly AmED usage. This suggests that high-dose habitual consumers of AmED are more vulnerable to ARPG. Consequently, identifying youth who engage in AmED use may be a useful screening indicator for individuals who may be at higher risk for problem gambling.

The present study's findings are subject to several limitations. Data were cross-sectional, thus, causal inferences cannot be drawn. Future research needs to assess the temporal relationship between AmED use and gambling to determine the causal pathways between AmED consumption and gambling. Self-reported data may have been biased by standard limitations (e.g., memory recall biases, social desirability, etc.). However, previous research has indicated that use of selfreported drinking is reliable and valid (Del Boca \& Darkes, 2003) and that most studies examining adolescent gambling use the SOGS-RA as the primary instrument to assess problem gambling (Edgren et al., 2016). Additionally, the time periods considered for the present study are easily remembered (e.g., AmED in the past 30 days) and data were collected anonymously. Energy drinks can vary significantly in their content, which makes having a standard drink measure or an objective measure of caffeine essential in this field of research. Furthermore, important indicators of consuming AmED combined with gambling problems (e.g., personality variables, decision-making processes) were not assessed in this study and such factors might have been important. However, the present study also has many strengths. The sample size was large and the data were nationally representative (of Italian youth). The study uniquely captured data regarding AmED and problem gambling (as measured by using the SOGS-RA) whereas previous studies have not collected data on adolescent problem gambling. 


\section{CONCLUSION}

The present study clearly established that consuming energy drink and alcohol simultaneously might pose significantly greater risk to experiencing gambling-related problems among adolescents. The findings suggest that policymakers and health care professionals should be aware that adolescents who consume AmED appear to be more prone to ARPG. For this reason, an avenue for future gambling-related prevention is to consider that caffeinated alcoholic beverage consumption acts as a potential risk factor for adolescent risky gambling. As heavier AmED consumption is linked to ARPG, a widely recognized impulsive behavior, self-regulation programs may be beneficial in reducing negative consequences experienced by this high-risk group. 


\section{REFERENCES}

Arria, A.M., \& O'Brien, M.C. (2011). The "high" risk of energy drinks. JAMA, 305(6), 600-601. DOI:10.1001/jama.2011.109.

Auger, N., Lo, E., Cantinotti, M., \& O'Loughlin, J. (2010). Impulsivity and socio- economic status interact to increase the risk of gambling onset among youth. Addiction, 105(12), 2176-2183. DOI: 10.1111/j.1360-0443.2010.03100.x.

Blaszczynski, A., \& Nower, L. (2002). A pathways model of problem and pathological gambling. Addiction, 97(5), 487-499. DOI:10.1046/j.1360-0443.2002.00015.x

Bonar, E. E., Cunningham, R. M., Polshkova, S., Chermack, S.T., Blow, F. C., \& Walton, M. A. (2015). Alcohol and energy drink use among adolescents seeking emergency department care. Addictive Behaviors, 43, 11-17. DOI:10.1016/j.addbeh.2014.11.023.

Brache, K., \& Stockwell, T. (2011). Drinking patterns and risk behaviors associated with combined alcohol and energy drink consumption in college drinkers. Addictive Behaviors, 36(12), 1133 1140. DOI:10.1016/j.addbeh.2011.07.003.

Brevers, D., Bechara, A., Cleeremans, A., \& Noël, X. (2013). Iowa Gambling Task (IGT): twenty years after-gambling disorder and IGT. Frontiers in Psychology, 4, 665. DOI:10.3389/fpsyg.2013.00665.

Canale, N., Scacchi, L., \& Griffiths, M. D. (2016). Adolescent gambling and impulsivity: Does employment during high school moderate the association? Addictive Behaviors, 60, 37-41. DOI:10.1016/j.addbeh.2016.04.001.

Canale, N., Vieno, A., Billieux, J., Lazzeri, G., Lemma, P., \& Santinello, M. (2017a). Is medicine use for nervousness associated with adolescent at-risk or problem gambling? European addiction research, 23(4), 171-176. DOI: 10.1159/000479001.

Canale, N., Vieno, A., Lenzi, M., Griffiths, M. D., Borraccino, A., Lazzeri, G., Scacchi, L., \& Santinello, M. (2017b). Income inequality and adolescent gambling severity: Findings from a 
large-scale Italian representative survey. Frontiers in Psychology, 8, 1318. DOI:10.3389/fpsyg.2017.01318.

Carbonneau, R., Vitaro, F., Brendgen, M., \& Tremblay, R.E. (2015). Trajectories of gambling problems from mid-adolescence to age 30 in a general population cohort. Psychology of Addictive Behaviors, 29(4), 1012-21. DOI:10.1037/adb0000102.

Colasante, E., Gori, M., Bastiani, L., Scalese, M., Siciliano, V., \& Molinaro, S. (2014). Italian Adolescent Gambling Behaviour: Psychometric Evaluation of the South Oaks Gambling Screen: Revised for Adolescents (SOGS-RA) among a sample of Italian students. Journal of Gambling Studies, 30(4), 789-801. DOI:10.1007/s10899-013-9385-6.

DAP-Italian Department of Anti-Drug Policies of the Presidency of the Council of Ministers. The 2016 Annual Report to Italian Parliament on the state of drug addiction. Retrieved February 15, 2017, from: http://www.politicheantidroga.gov.it/media/1095/1-relazione-annuale-alparlamento-2016-sullo-stato-delle-tossicodipendenze-in-italia.pdf.

Del Boca, F.K., \& Darkes, J. (2003). The validity of self-reports of alcohol consumption: state of the science and challenges for research. Addiction, 98(Suppl 2), 1-12. DOI:10.1046/j.13596357.2003.00586.x.

Derevensky, J.L., \& Gilbeau, L. (2015). Adolescent gambling: twenty-five years of research. Canadian Journal of Addiction, 6(2), 4-12.

Diller, J. W., Saunders, B. T., Anderson, K. G. (2008). Effects of acute and repeated administration of caffeine on temporal discounting in rats. Pharmacology, Biochemistry and Behavior, 89(4), 546-555. doi: 10.1016/j.pbb.2008.02.00

Dowling, N. A., Merkouris, S. S., Greenwood, C. J., Oldenhof, E., Toumbourou, J. W., Youssef, G. J. (2017). Early risk and protective factors for problem gambling: A systematic review and meta-analysis of longitudinal studies. Clinical Psychology Review, 51, 109-124. DOI: 10.1016/j.cpr.2016.10.008. 
Dussault, F., Brendgen, M., Vitaro, F., Wanner, B., \& Tremblay, R. E. (2011). Longitudinal links between impulsivity, gambling problems and depressive symptoms: a transactional model from adolescence to early adulthood. Journal of Child Psychology and Psychiatry, 52(2), 130-38. DOI:10.1111/j.1469-7610.2010.02313.x.

Edgren, R., Castrén, S., Mäkelä, M., Pörtfors, P., Alho, H., Salonen, A.H. (2016). Reliability of instruments measuring at-risk and problem gambling among young individuals: A Systematic review covering years 2009-2015. Journal of Adolescent Health, 58(6), 600-615. DOI:10.1016/j.jadohealth.2016.03.007.

FDA News Release. FDA warning letters issued to four makers of caffeinated alcoholic beverages. Nov 2010. Retrieved February 15, 2017, from: http://www.fda.gov/NewsEvents/Newsroom/PressAnnouncements/ucm234109.htm. Accessed

Ferris, J., \& Wynne, H. (2001). The Canadian Problem Gambling Index: Final Report. Toronto, ON: Canadian Centre on Substance Abuse.

Flora, S., \& Dietze, M. (1993). Caffeine and impulsivity in rats. Bulletin of the Psychonomic Society, 31, 39-41.

Fuxe, K., Ferré, S., Genedani, S., Franco, R., \& Agnati, L. F. (2007). Adenosine receptor-dopamine receptor interactions in the basal ganglia and their relevance for brain function. Physiology and Behavior, 92(1-2), 210-217. DOI:10.1016/j.physbeh.2007.05.034.

Grant, J. E., Odlaug, B. L., \& Chamberlain, S. R. (2016). Neural and psychological underpinnings of gambling disorder: a review. Progress in Neuropsychopharmacology and Biological Psychiatry, 65, 188-193. DOI:10.1016/j.pnpbp.2015.10.007.

Hayer, T., \& Griffiths, M. D. (2015). Gambling. In Gullotta, T. P., Plant, R., W., Evans, \& M. A. (Eds). Handbook of adolescent behavioral problems: Evidence based approaches to prevention and treatment (2nd ed.) (pp. 539 -558). New York, NY: Springer. 
Leeman, R. F., \& Potenza, M. N. (2012). Similarities and differences between pathological gambling and substance use disorders: A focus on impulsivity and compulsivity. Psychopharmacology (Berl), 219(2), 469-490. DOI:10.1007/s00213-011-2550-7.

Lyvers, M. (2000). "Loss of control" in alcoholism and drug addiction: a neuroscientific interpretation. Experimental and Clinical Psychopharmacology, 8(2), 225-249. DOI:10.1037/1064-1297.8.2.225.

Marczinski, C. A. (2011). Alcohol mixed with energy drinks: consumption patterns and motivations for use in US college students. International Journal of Environmental Research and Public Health, 8(8), 3232-3245. DOI:10.3390/ijerph8083232.

McKetin, R., Coen, A., \& Kaye, S. (2015). A comprehensive review of the effects of mixing caffeinated energy drinks with alcohol. Drug and Alcohol Dependence, 151, 15-30. DOI:10.1016/j.drugalcdep.2015.01.047.

Miller, K. E. (2008). Energy drinks, race, and problem behaviors among college students. Journal of Adolescent Health, 43(5), 490-497. DOI:10.1016/j.jadohealth.2008.03.003.

Molinaro, S., Canale, N., Vieno, A., Lenzi, M., Siciliano, V., Gori, M., \& Santinello, M. (2014). Country- and individual level determinants of probable problematic gambling in adolescence: A multi-level cross-national comparison. Addiction, 109(12), 2089-2097. DOI: 10.1111/add.12719.

Peacock, A., Pennay, A., Droste, N., Bruno, R., \& Lubman, D. (2014). "High" risk? A systematic review of the acute outcomes of mixing alcohol with energy drinks. Addiction, 109(10), 16121633. DOI:10.1111/add.12622.

Peacock, A., Droste, N., Pennay, A., Lubman, D.I., Miller, P., Newcombe, D., \& Bruno, R. (2015). Self-reported risk-taking behavior during matched-frequency sessions of alcohol versus combined alcohol and energy drinks consumption: does co-ingestion increase risk-taking? Alcoholism, Clinical and Experimental Research, 39(5), 911-8. DOI:10.1111/acer.12700. 
Pennay, A., Cheetham, A., Droste, N., Miller, P., Lloyd, B., Pennay, D., Dowling, N., Jackson, A., \& Lubman, D.I. (2015). An examination of the prevalence, consumer profiles, and patterns of energy drink use, with and without alcohol, in Australia. Alcoholism, Clinical and Experimental Research, 39(8), 1485-92. DOI:10.1111/acer.12764.

Polak, K., Dillon, P., Koch, J.R., Miller, W.G.Jr, Thacker. L., \& Svikis, D. (2016). Energy drink use is associated with alcohol and substance use in eighth, tenth, and twelfth graders. Preventive Medicine Reports, 4, 381-4. DOI:10.1016/j.pmedr.2016.06.019.

Potenza, M. N. (2014). The neural bases of cognitive processes in gambling disorder. Trends in Cognitive Sciences, 8(8), 429-438. DOI:10.1016/j.tics.2014.03.007.

Robins, M. T., Lu, J., \& van Rijn, R. M. (2016). Unique behavioral and neurochemical effects induced by repeated adolescent consumption of caffeine-mixed alcohol in C57BL/6 mice. PloS One,11(7), e0158189. DOI:10.1371/journal.pone.0158189.

Scalese, M., Denoth, F., Siciliano, V., Bastiani, L., Cotichini, R., Cutilli, A., \& Molinaro, S. (2017). Energy drink and alcohol mixed energy drink use among high school adolescents: association with risk taking behavior, social characteristics. Addictive Behaviors, 72, 93-99. DOI:10.1016/j.addbeh.2017.03.016.

Seifert, S. M., Schaechter, J. L., Hershorin, E. R., \& Lipshultz, S. E. (2011). Health effects of energy drinks on children, adolescents, and young adults. Pediatrics, 127(3), 511-528. DOI:10.1542/peds.2009-3592.

Snipes, D. J., Jeffers, A. J., Green, B. A., \& Benotsch, E. G. (2015). Alcohol mixed with energy drinks are robustly associated with patterns of problematic alcohol consumption among young adult college students. Addictive Behaviors, 41, 136-141. DOI:10.1016/j.addbeh.2014.10.010.

Steinberg, L., Mounts, N.S., Lamborn, S.D., \& Dornbusch, S.M. (1991). Authoritative parenting and adolescent adjustment across varied ecological niches. Journal of Research on Adolescence, 1(1), 19-36. DOI:10.1111/1532-7795.ep11522650. 
Striley, C. W., \& Khan, S. R. (2014). Review of the energy drink literature from 2013: findings continue to support most risk from mixing with alcohol. Current Opinion in Psychiatry, 27(4), 263-268. DOI:10.1097/YCO.0000000000000070.

van Nieuwenhuijzen, M., Junger, M., Velderman, M. K., Wiefferink, K. H., Paulussen, T. W., Hox, J., \& Reijneveld, S. A. (2009). Clustering of health-compromising behavior and delinquency in adolescents and adults in the Dutch population. Preventive Medicine, 48(6), 572-578. DOI:10.1016/j.ypmed.2009.04.008.

Walther, B., Morgenstern, M., \& Hanewinkel, R. (2012). Co-occurrence of addictive behaviours: Personality factors related to substance use, gambling and computer gaming. European Addiction Research,18(4), 167-74. DOI:10.1159/000335662.

Wardell, J. D., Quilty, L. C., Hendershot, C. S., Bagby, R. M. (2015). Motivational pathways from reward sensitivity and punishment sensitivity to gambling frequency and gambling-related problems. Psychology of Addictive Behaviors, 29(4), 1022-1030. DOI:10.1037/adb0000066.

Welte, J. W., Barnes, G. M., Tidwell, M. C. O., \& Hoffman, J. H. (2008). The prevalence of problem gambling among US adolescents and young adults: Results from a national survey. Journal of Gambling Studies, 24(2), 119-133. DOI:10.1007/s10899-007-9086-0.

Winters, K. C., Stinchfield, R. D., \& Fulkerson, J. (1993). Toward the development of an adolescent gambling problem severity scale. Journal of Gambling Studies, 9(1), 63-84. DOI:10.1007/BF01019925.

Zucconi, S., Volpato, C., Adinolfi, F., \& Gandini, E. (2013). Gathering consumption data on specific consumer groups of energy drinks. European Food Safety Authority. Supporting Publications: EN-394. Retrieved April 15, 2017, from: http:// www.efsa.europa.eu/publications. Zuckerman, M. (1994). Behavioral expressions and biosocial bases of sensation seeking. Cambridge: Cambridge University Press. 


\section{Figure legends}

Figure 1. Prevalence of at-risk/problem gambling (during the past 12 months) by beverage consumption

Figure 2. Adjusted Odd ratios (aORs) by beverage consumption ["no beverage consumption" ref category]

Note: Black boxes show the relative risk, with box size indicating the precision of the estimate (bigger size refers to more precision). Orange lines indicate $95 \%$ confidence intervals. 


\section{Tables}

Table 1. Percentage (\%) of participant's characteristics

Total $(n=4,495)$

Beverage consumption last month (LM) [ $\geq 6$ time]

No

Alcohol alone

71.7

AmED

23.2

5.1

Beverage consumption LM [ $\geq 10$ time $]$

No

Alcohol alone

13.0

AmED

2.7

Beverage consumption LM [ $\geq 20$ time]

No

Alcohol alone

4.8

AmED

1.6

Gender

Girls

37.5

Boys

62.5

Age

15-17 years

52.1

18-19 years

47.9

Parent's Education

$4.71(1.12)^{\#}$

Perceived economic family status

High

24.1

Medium

66.0

Low

9.0

Gambling severity

No problem gambling

81.0

At risk/problem gambling (ARPG) 19.0

"M(SD); "Completed primary school or less" = 1, "some secondary school" = 2, "completed secondary school" = 3, "some college" = 4, "completed college or some university" $=5$, "completed university" $=6$ 
Table 2. ORs $(95 \% \mathrm{CI})$ for ARPG $(0=$ non-problem gamblers, $1=$ at risk-problem gamblers $)$ according to AmED and alcohol alone consumption (Model 1), adjusted ORs (aORs) for gender, age, parent's education and perceived economic family status (Model 2)

At risk/problem gambling [ARPG]

\begin{tabular}{ll}
\hline Model 1 & Model 2 \\
ORs $(95 \% \mathrm{CI})$ & aORs $(95 \% \mathrm{CI})$
\end{tabular}

Beverage consumption LM [>=6 time]

No

Alcohol alone

AmED
Ref

$1.66(1.39-1.97)^{* * *}$

$4.19(3.17-5.53)^{* * *}$
Ref

$1.37(1.14-1.65)^{* *}$

$3.05(2.24-4.16)^{* * *}$

Gender

Girls

Ref

Boys

$3.74(3.04-4.59) * * *$

Age

15-17 years

Ref

18-19 years

$1.06(.90-1.25)$

Parent's Education

$0.88(.83-.95)^{* *}$

Perceived economic family status

High

Ref

Medium

$.82(.67-.99)^{*}$

Low

$1.10(.81-1.48)$

$* \mathrm{p}<.05 ; * * \mathrm{p}<.01 ; * * * \mathrm{p}<.001$ 